\title{
PaRoT: A Practical Framework for Robust Deep Neural Network Training
}

\author{
Edward W. Ayers ${ }^{1}$, Francisco Eiras $^{2}$, Majd Hawasly ${ }^{2}$, Iain Whiteside ${ }^{2}$ \\ 1 DPMMS, Cambridge University, United Kingdom, e.w. ayers@maths.cam.ac.uk \\ 2 FiveAI, 20 Cambridge Place, Cambridge, United Kingdom, first.last@five. ai
}

\begin{abstract}
Deep Neural Networks (DNNs) are finding important applications in safety-critical systems such as Autonomous Vehicles (AVs), where perceiving the environment correctly and robustly is necessary for safe operation. Raising unique challenges for assurance due to their black-box nature, DNNs pose a fundamental problem for regulatory acceptance of these types of systems. Robust training — training to minimize excessive sensitivity to small changes in input — has emerged as one promising technique to address this challenge. However, existing robust training tools are inconvenient to use or apply to existing codebases and models: they typically only support a small subset of model elements and require users to extensively rewrite the training code. In this paper we introduce a novel framework, PaRoT, developed on the popular TensorFlow platform, that greatly reduces the barrier to entry. Our framework enables robust training to be performed on existing DNNs without rewrites to the model. We demonstrate that our framework's performance is comparable to prior art, and exemplify its ease of use on off-the-shelf, trained models and its testing capabilities on a realworld industrial application: a traffic light detection network.
\end{abstract}

\section{Introduction}

Deep Neural Networks (DNNs) are finding important applications in safety-critical systems, such as Autonomous Vehicles (AVs), where perceiving a complex environment correctly and robustly is necessary for safe operation $[17,11,4]$. The challenge of assuring these so-called AI-enabled systems is well-known [22] and has attracted the attention of researchers and research bodies, e.g., DARPA [9]. Existing standards and techniques - such as the ubiquitous ' $\mathrm{V}$ ' model - lean heavily on the existence of a clear specification to verify against [32]. Unfortunately, the very nature of deep learning - where the specification is implicit in the training data — poses a fundamental problem for regulatory acceptance of these systems in a safety-critical domain.

One of the most troubling features of DNNs is their 'intriguing' susceptibility to adversarial examples: imperceptible perturbations in the input space that cause a large change in the output space. For example, causing an object detection network to misclassify an image [35]. Figure 1 shows an adversarial example on a traffic light detector.

The formal verification community has responded to this provocation with gusto [20, $19,13,3,16,5]$. Exacerbating the verification challenge is the indirect nature of any 'fixes' that can be applied to failure of post-hoc formal verification for a DNN: typically 


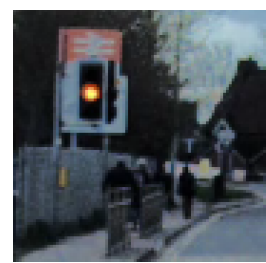

(a)

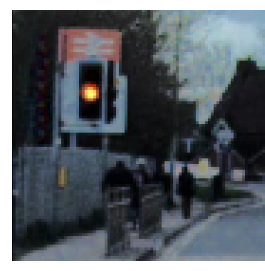

(b)

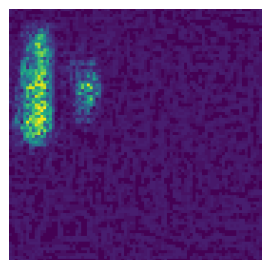

(c)

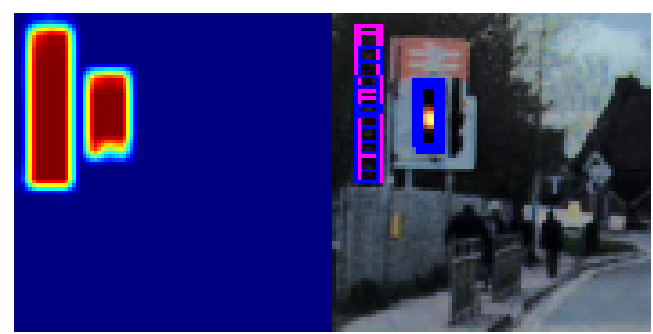

(d)

Fig. 1. Traffic Light Detection Network: (a) an image from the test set in which the traffic light is identified correctly; (b) an adversarial example: a subtly modified version of the original image, identified using PaRoT; (c) norm of the difference between the original image and the adversarial images; (d) the inference result on the adversarial example, with a confidence heatmap on the left and bounding boxes of the identified traffic lights on the right.

an augmentation to the training set. Unlike with traditional software, fixes to DNNs can feel very much like playing a game of whack-a-mole.

The emerging robust training paradigm, which integrates the verification process directly into the training scheme, is, in our view, the most promising approach towards formally verified neural networks. The goal of robust training is to minimize a so-called worst-case adversarial loss. Formally, let $N_{\theta}: \mathbb{R}^{p} \rightarrow \mathbb{R}^{q}$ be a neural network with $p$ input features and $q$ outputs, parameterized with weights $\theta$. Let $\mathcal{B}_{\epsilon}(x)$ be an $\ell_{\infty}$-ball of radius $\epsilon$ around an input point $x \in \mathbb{R}^{p}$. For a given loss function $\mathcal{L}$, we can define the worst-case adversarial loss $\mathcal{L}_{N_{\theta}}$ at a point $x$ as:

$$
\mathcal{L}_{N_{\theta}}(x, y):=\max _{\tilde{x} \in \mathcal{B}_{\epsilon}(x)} \mathcal{L}\left(N_{\theta}(\tilde{x}), y\right)
$$

In general, one may replace the ball $\mathcal{B}_{\epsilon}(x)$ with some parameterized set $\pi_{\epsilon}(x)$. For a set of labelled training data $\left\{\left(x_{i}, y_{i}\right)\right\}_{i=1}^{n}$, robust training can be formulated as a saddlepoint problem:

$$
\min _{\theta} \max _{i} \mathcal{L}_{N_{\theta}}\left(x_{i}, y_{i}\right)
$$

Finding the worst-case adversarial loss for a given example is computationally expensive in general. In practice, most approaches approximate the worst-case adversarial loss in one way or another $[41,42,27]$. In recent years, robust training has progressed from single layer, dense networks to moderate - though not yet state-of-the-art - sized Convolutional Neural Networks (CNNs). This has brought these techniques 
within the realm of various DNNs used within the reference AV stack being built by Five AI. In our bid to understand the practicalities of robust training, we found that existing tools are inconvenient to use or apply to existing models: they typically only support a small subset of model elements and require users to re-specify the models in a specialized language, which can mean extensive rewrites to the training code.

To tackle these problems, we introduce a framework in this paper, called Practical Robust Training $(\mathrm{PaRoT})^{1}$, developed on the popular TensorFlow platform [1]. Our framework allows robust training — using differentiable abstract interpretation [27] — to be performed on arbitrary DNNs without any rewrites of the model. In PaRoT, one can start a robust model training for a popular convolutional neural network with a minimal amount of code, as we demonstrate in Listing 1.2. We have, for example, used PaRoT to robustly train the traffic light detection network seen in Figure 1.

Contributions The main contribution of this paper is a practical framework, PaRoT, built in the Tensorflow platform [1]. In particular,

- Our tool can automatically apply abstract interpretation on an existing model definition. Thus, it can be used to verify robustness on existing DNNs without having to change the model code, allowing for seamless adoption with existing codebases.

- Our framework implements a broad set of robustness properties that go beyond the usual $\epsilon$-ball, and provides a clean interface for specifying custom properties.

- We improve upon the abstract interpretation techniques used by Mirman et al. [27]. In particular, we refine several abstract transformers for activation functions.

Structure of paper In Section 2 we introduce the requisite background in robust training with abstract interpretation. In Section 3 we describe the architecture and functionality of the PaRoT framework and evaluate its performance in Section 4. In Section 5 we place our work more broadly in the field of formal verification of DNNs. Finally, in Section 6, we conclude and present future directions for this framework and paradigm.

\section{Background}

We build on the robust training approach of DiffAI, introduced by Mirman et al. [27], where the inner maximization of Equation 2 is approximated using abstract interpretation. In this section, we sketch the mathematical prerequisites to our framework.

\subsection{Abstract Interpretation}

Abstract interpretation is a general theory for approximating infinite sets of behaviours with a finite representation [7,8]. In the present study, this corresponds to convex approximations of a non-convex adversarial polytope.

The two basic constructs in abstract interpretation are the abstract domain and the abstract transformer. Intuitively, an abstract domain gives a finite (approximate) representation of the (potentially infinite) concrete space, while an abstract transformer

\footnotetext{
${ }^{1}$ The framework is available at https://github.com/fiveai/parot
} 


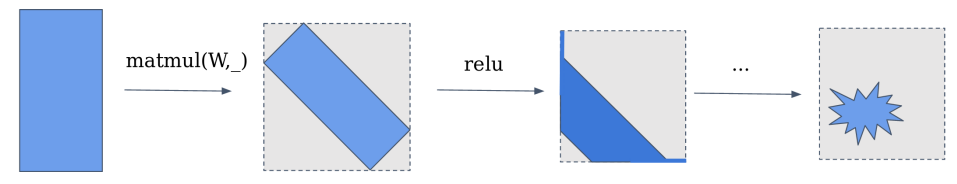

Fig. 2. An illustration of abstract domains. The dotted grey box corresponds to a domain object, and the blue shape is the true set that the domain object seeks to approximate.

provides an over-approximation of the behaviour of a function. Formally, an abstract domain is a set $\mathcal{D}$ (the domain) and a pair of maps $\alpha: \mathcal{P}\left(\mathbb{R}^{p}\right) \rightarrow \mathcal{D}$ and $\gamma: \mathcal{D} \rightarrow \mathcal{P}\left(\mathbb{R}^{p}\right)$, called the abstraction and concretization maps, respectively. $\mathcal{P}(X)$ is the powerset of $X$. The abstraction function is defined such that $U \subseteq \gamma(\alpha(U))$ for all $U \subseteq \mathbb{R}^{p}$.

Additionally, an abstract domain is equipped with a mapping from a fixed set of primitive functions $\mathcal{F}$ to abstract transformers in $\mathcal{D}$ such that each $f: \mathbb{R}^{p} \rightarrow \mathbb{R}^{q}$ in $\mathcal{F}$ is mapped to a function $\mathcal{D}(f): \mathcal{D} \rightarrow \mathcal{D}^{\prime}$. For each element in the concrete space, $z$, transformers must obey the following soundness relation:

$$
f[\gamma(z)] \subseteq \gamma(\mathcal{D}(f)(z))
$$

This ensures that transformers produce new abstract elements whose concretization overapproximates the image of the function. Since transformers compose, we may transform any composite function $f=f_{1} \circ f_{2} \circ \cdots \circ f_{n}: \mathbb{R}^{p} \rightarrow \mathbb{R}^{q}$ where $f_{i} \in \mathcal{F}$. Figure 2 illustrates graphically the abstract domains and transformers for a single layer of a DNN. We can construct a composite transformer $\mathcal{D}(N)$ that represents that network, and write the sound approximation for an $\epsilon$-ball around a point $x$ as:

$$
\gamma\left(\mathcal{D}(N)\left[\alpha\left(\mathcal{B}_{\epsilon}(x)\right)\right]\right)
$$

\subsection{Abstract Domains for DNNs}

We consider three abstract domain types: BOX, ZONOTOPE and HYBRIDZONOTOPE:

- Box, represented by $i=\langle\mathbf{c}, \mathbf{b}\rangle$. A Box domain is a $p$-dimensional axis-aligned box, parameterized by its center $\mathbf{c} \in \mathbb{R}^{p}$ and a positive vector $\mathbf{b} \in \mathbb{R}_{>0}^{p}$ containing the half-widths of the box. Figure 2 illustrates the concept of the Box domain.

- ZONOTOPE, represented by $z=\langle\mathbf{c}, \mathbf{E}\rangle$. For dimension $p$, a ZONOTOPE is parameterized by a center point $\mathbf{c} \in \mathbb{R}^{p}$ as well as a matrix $\mathbf{E} \in \mathbb{R}^{p \times e}$ for some fixed dimension $e$. The set $z \subseteq \mathbb{R}^{p}$ is the $\mathbf{E}$ image of an $e$-dimensional hypercube, centerd at $\mathbf{c}$. The concretization is given by:

$$
\gamma(z):=\left\{\mathbf{c}+\mathbf{E} \mathbf{v}:\left|\mathbf{v}_{i}\right| \leq 1, i \in\{1, \ldots, e\}\right\}
$$

The key feature of a ZONOTOPE domain is that transformers exist for affine functions - such as the matrix multiplications associated with transition functions of DNNs - that do not increase the approximation error. 
- HybridZonotope, represented by $h=\langle\mathbf{c}, \mathbf{b}, \mathbf{E}\rangle$. One problem with the ZonoTOPE domain is that computation can be expensive compared to a BOX domain. The HYBRIDZONOTOPE solves this problem with the inclusion of an extra positive vector $\mathbf{b} \in \mathbb{R}_{>0}^{p}$, with a concretization:

$$
\begin{aligned}
\gamma(h):=\left\{\mathbf{c}+\mathbf{E} \mathbf{v}+\operatorname{diag}(\mathbf{b}) \mathbf{w}|| \mathbf{v}_{i} \mid \leq 1,\right. & \left|\mathbf{w}_{j}\right| \leq 1, \\
& i, \in\{1, \ldots, e\}, j \in\{1, \ldots, p\}\} .
\end{aligned}
$$

Note that these definitions mean that BOX and ZONOTOPE are both subsets of HYBRIDZONOTOPE. In the HYBRIDZONOTOPE domain, it is possible to convert $\mathbf{b}$ values to $\mathbf{E}$ values and vice-versa through correlation and decorrelation, as noted in [28].

\subsection{Hybrid Zonotope Transformers for DNNs}

It is straightforward to show that exact transformers can be constructed for matrix multiplication [27]. In contrast, accurate modeling of piecewise linear activation functions, such as relu $(x):=\max (x, 0)$, necessarily introduce an approximation. Here we generalize the work in [34] to find optimal hybrid zonotopes for a given activation function. Since activations are one-dimensional (1D) and act on each dimension separately, we may consider just the problem in 1D. For a given function $f: \mathbb{R} \rightarrow \mathbb{R}$ and input bounds $\underline{x}, \bar{x}$, the challenge is to find a parallelogram containing the graph of $f$ restricted to $[\underline{x}, \bar{x}]$ that has minimal area, as shown in Figure 3 below.

In the first instance, we consider an activation function $f$ which is convex or concave. If $\bar{x}=\underline{x}$, we can treat the transformer as acting on a point. Otherwise, we compute the slope of the parallelogram:

$$
\mu:=\frac{f(\bar{x})-f(\underline{x})}{\bar{x}-\underline{x}}
$$

We provide an extremum function $x_{f}(\mu)$ for the given $f$. Assuming a convex function:

$$
x_{f}(\mu)=\underset{x \in \mathbb{R}}{\operatorname{argmin}}(f(x)-\mu x)
$$

If $f$ is concave, replace argmin with argmax. Since $f$ is convex/concave, this $x_{f}(\mu)$ will always be in the interval $[\underline{x}, \bar{x}]$ or otherwise $f(x)-\mu x$ is zero everywhere in $[\underline{x}, \bar{x}]$. For many of the activation functions we care about, it is simple to find these extremum functions. For example, $x_{\text {relu }}(\mu)=0$ and $x_{\exp }(\mu)=\ln \mu$. Then, one can compute:

$$
e:=x_{f}(\mu) \cdot \mu-\frac{f(\underline{x}) \cdot \bar{x}+\underline{x} \cdot f(\bar{x})}{\bar{x}-\underline{x}}
$$

which may be interpreted as the height of the resulting zonotope parallelogram. From this we may compute the center of the parallelogram in the $y$ direction:

$$
c_{y}:=\frac{1}{2}(f(\bar{x})+f(\underline{x})-e)
$$

Finally we compute the new 1D hybrid zonotope: 


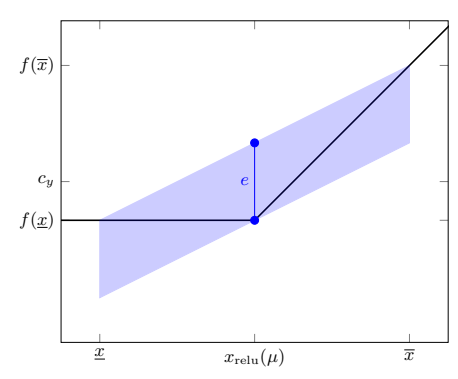

(a)

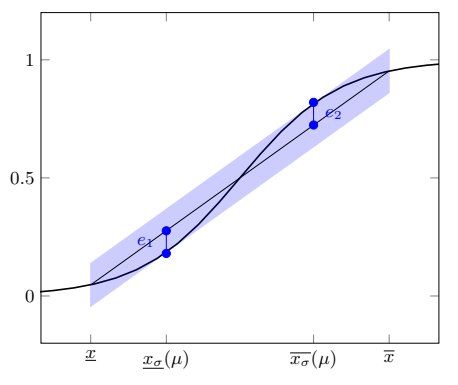

(b)

Fig. 3. Constructing zonotope transformers for relu and sigmoid activation functions.

$$
D(f)\left\langle c_{x}, b_{x}, \mathbf{E}_{x}\right\rangle=\left\langle c_{y}, \mu b_{x}+\frac{e}{2}, \mu \mathbf{E}\right\rangle
$$

To extend this approach to nonconvex functions, such as sigmoid, we instead need to find a pair of extrema $x_{f}(\mu), \overline{x_{f}}(\mu)$ which may in general depend on the interval bounds $[\underline{x}, \bar{x}]$. In the case of sigmoid, one can show that these are minus the natural logarithm of the solutions $Y_{ \pm}$to the quadratic equation $\mu+(2 \mu-1) Y+\mu Y^{2}=0$. Figure 3 shows zonotope transformers for relu and sigmoid activation functions.

\subsection{Robust Training}

To train with an abstract domain on a model $N$, from each training datum $(\mathbf{x}, \mathbf{y})$ we compute a prediction value $N(\mathbf{x})$ and a transformed domain object $\mathcal{D}(N)\left(\mathcal{B}_{\epsilon}(\mathbf{x})\right)$ of the domain representation of an $\ell_{\infty}$-ball $\mathcal{B}_{\epsilon}(\mathbf{x})$ around the input $\mathbf{x}$ for some fixed perturbation radius $\epsilon$. An axis-aligned bounding box is drawn around the resulting output domain object, and the vertex $v$ furthest away from the true target $\mathbf{y}$ is chosen. We construct a combined loss $\mathcal{L}_{\text {comb }}$ with the standard loss, the adversarial loss, a mixing factor $\lambda \in \mathbb{R}_{\geq 0}$, and a regularization term $\xi(N)$ :

$$
\mathcal{L}_{\text {comb }}(\mathbf{x}, \mathbf{y}):=\mathcal{L}(N(\mathbf{x}), \mathbf{y})+\lambda \mathcal{L}\left(\underset{\mathbf{v} \in \mathcal{D}(N)(\mathbf{x})}{\operatorname{argmax}}\|\mathbf{v}-\mathbf{y}\|_{2}, \mathbf{y}\right)+\xi(N)
$$

\section{PaRoT System Description}

In this section, we detail how PaRoT can be used for robust training and testing. The main overview of the system is presented in Figure 4. The training aspects of the framework can be divided into domains (in the module parot . domains), which correspond to the ones identified in Section $2.2^{3}$, and properties (in the module parot.properties) corresponding to the types of adversaries we are trying to robustify against. Section 3.2 presents the built-in properties available in PaRoT. As our system uses the TensorFlow platform, we first introduce some terminology.

\footnotetext{
${ }^{3}$ With the exception of the ZONOTOPE domain, which is not implemented in PaRoT.
} 


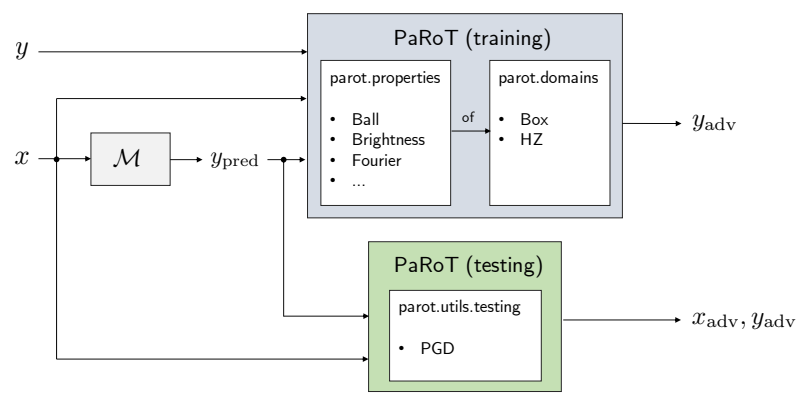

Fig. 4. PaRoT overview. Robust training is enabled by a property and an abstraction domain which can be chosen from those supported by PaRoT or extended with a custom domain. Given an input and a model prediction, PaRoT creates a domain object for the input based on the specified property, and it automatically transforms the operations associated with the model (see Section 3.1). At testing time, PaRoT provides auxiliary utilities.

TensorFlow [1] is a deep learning platform that enables the user to build a computation graph representing their neural network model and training scheme. This computation graph is a directed, acyclic graph whose nodes are tensors - a generalization of matrices to potentially higher dimensions - and whose edges are called ops and consist of a list of input and output tensors. An output tensor can be the input tensor for arbitrarily many ops. To illustrate, the left-hand side of Figure 5 shows the computation graph constructed for a single dense layer of a neural network. The ops MatMul (matrix multiplication), BiasAdd (adding a bias to a value), and ReLU (rectified linear unit operation) form those required to represent this example layer. Once a computation graph has been created, TensorFlow compiles it, allowing PaRoT to use this graph to automatically derive abstract transformers for a given model, as described in Section 3.1. This enables a user to use an existing model and immediately start robust training without needing code rewrites. It should be noted that the models supported by PaRoT must use only the operations supported by the framework in the selected domain. A list of the operations is available in Appendix A.

\subsection{Automatic Transformer Generation}

In order to transform a computation graph $G$ from a given input tensor $\mathrm{x}$ to an output tensor $\mathbf{z}$, we find the subgraph $S_{\mathbf{x}, \mathbf{z}}$ of $G$ whose vertices are the ys such that there exist paths $\mathbf{x} \rightsquigarrow \mathbf{y}$ and $\mathbf{y} \rightsquigarrow \mathbf{z}$. This can be easily extended to multiple inputs and outputs. This subgraph $S_{\mathbf{x}, \mathbf{z}}$ is found through a graph traversal algorithm backtracking from $\mathbf{z}$, which also produces a pair of adjacency maps $C$ and $M$. $C$ maps a tensor to a set of ops which consume it, while $M$ maps an op $f$ to the indices of the output tensors of the op in $G$. Once $S_{\mathbf{x}, \mathbf{z}}$ is constructed, the transformation process can begin. The output of the process is a dictionary $\mathcal{T}$ which maps $p$-dimensional tensors to domain objects $D$ (or the constant None). $\mathcal{T}$ is constructed by iteratively exploring $S_{\mathbf{x}, \mathbf{z}}$ starting at $\mathbf{x}$. The complete transformation algorithm is given in Algorithm 1. 


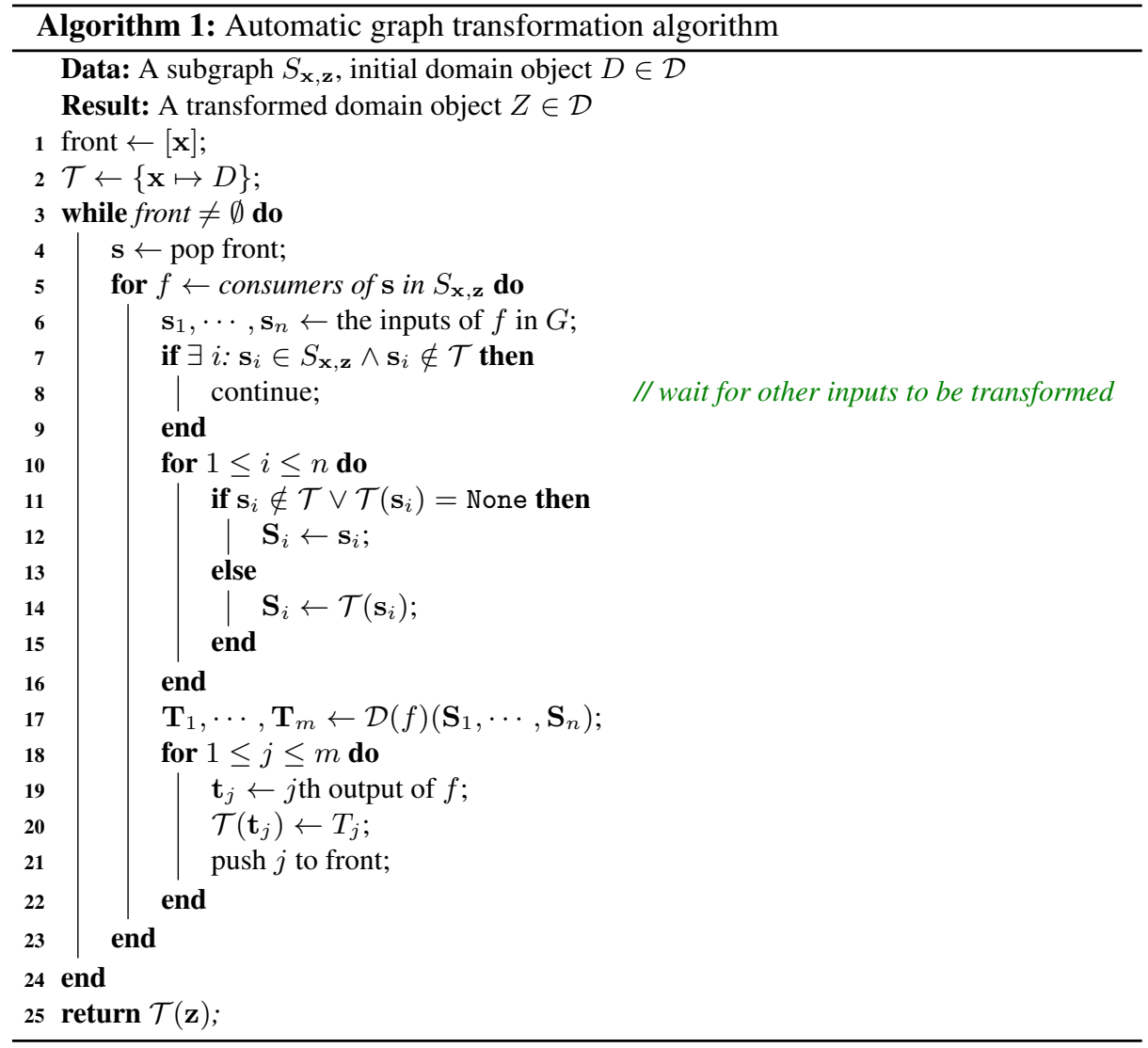

When transforming ops, various challenges arise. For example, a transformer $\mathcal{D}(f)$ can accept inputs that are not domain objects but instead just tensors. This occurs, for example, when a constant tensor needs to be added to a domain object. The acyclic graph structure makes this transformation non-trivial. The first issue arises when an operation consumes two or more domain objects. This happens in reticulated model architectures e.g., SkipNet from [40].

To illustrate the challenges of transforming ops, take two tensors $\mathbf{x}, \mathbf{y}$, consider the transformed computation graph for their addition $\mathbf{x}+\mathbf{y}$ where both $\mathbf{x}$ and $\mathbf{y}$ have abstract domains to be transformed. To transform + for the BoX domain, this entails merely adding the cs and $\mathbf{b s}$ of $\mathbf{x}$ and $\mathbf{y}$. However, for HYBRIDZONOTOPE, the manner with which the merging should take place depends on how the $\mathbf{E}$ matrices were constructed. If $\mathbf{x}$ and $\mathbf{y}$ are both derived from the same starting zonotope, then their $\mathbf{E}$ matrices will both be referencing the same parameterization. In this case the $\mathbf{E}$ matrices for $\mathbf{x}$ and $\mathbf{y}$ can be added. However, if they originate from different starting zonotopes, then their $e$ dimensions may not match up, and in this case they need to be concatenated 
along the $e$ dimension:

$$
\left\langle\mathbf{c}_{\mathbf{x}}, \mathbf{b}_{\mathbf{x}}, \mathbf{E}_{\mathbf{x}}\right\rangle+\left\langle\mathbf{c}_{\mathbf{y}}, \mathbf{b}_{\mathbf{y}}, \mathbf{E}_{\mathbf{y}}\right\rangle:=\left\langle\mathbf{c}_{\mathbf{x}}+\mathbf{c}_{\mathbf{y}}, \mathbf{b}_{\mathbf{x}}+\mathbf{b}_{\mathbf{y}},\left[\mathbf{E}_{\mathbf{x}}, \mathbf{E}_{\mathbf{y}}\right]\right\rangle
$$

Similar considerations must be made for, e.g., the Concat op which concatenates two tensors along a given dimension.

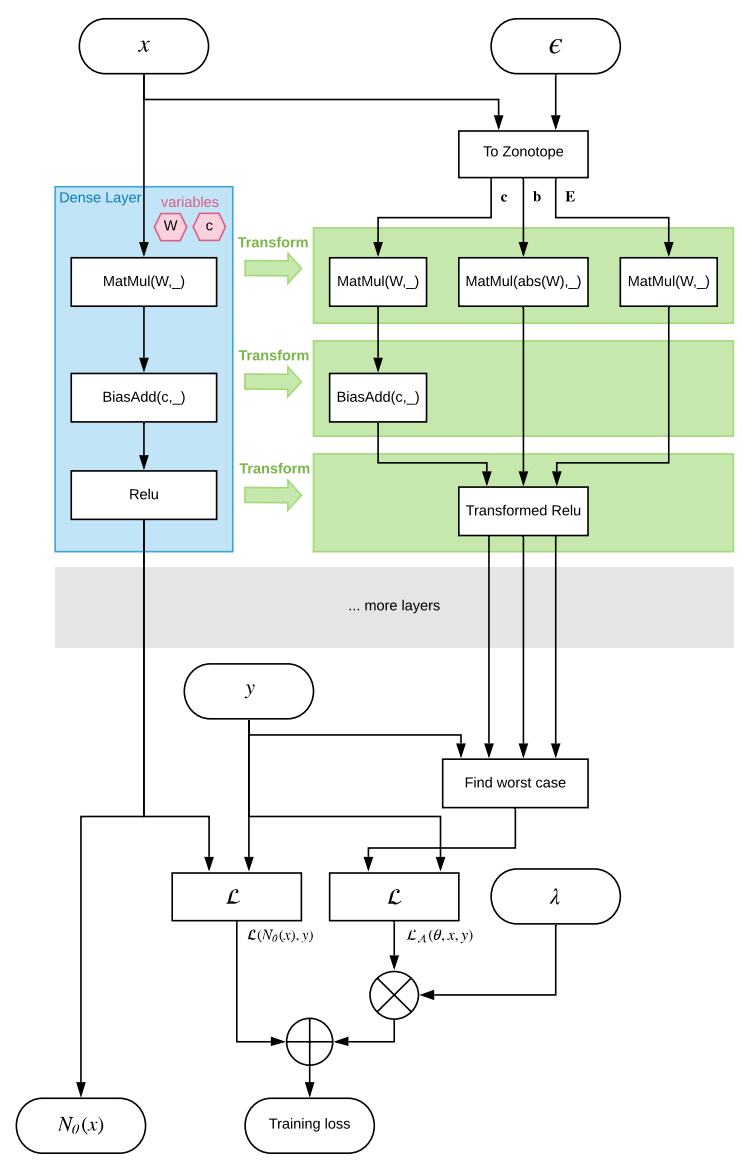

Fig. 5. An example computation graph for robust training showing the original (blue) and generated (green) computation graph on a dense layer.

Another complication in extending transformers to computation graphs arises with ops which do not output a transformed domain object. The most prominent example of this is the Shape op which returns the dimensions of a tensor. We support these operations by allowing the domain implementer to return None instead of a domain object, flagging that the transformer algorithm should use the un-transformed output. 


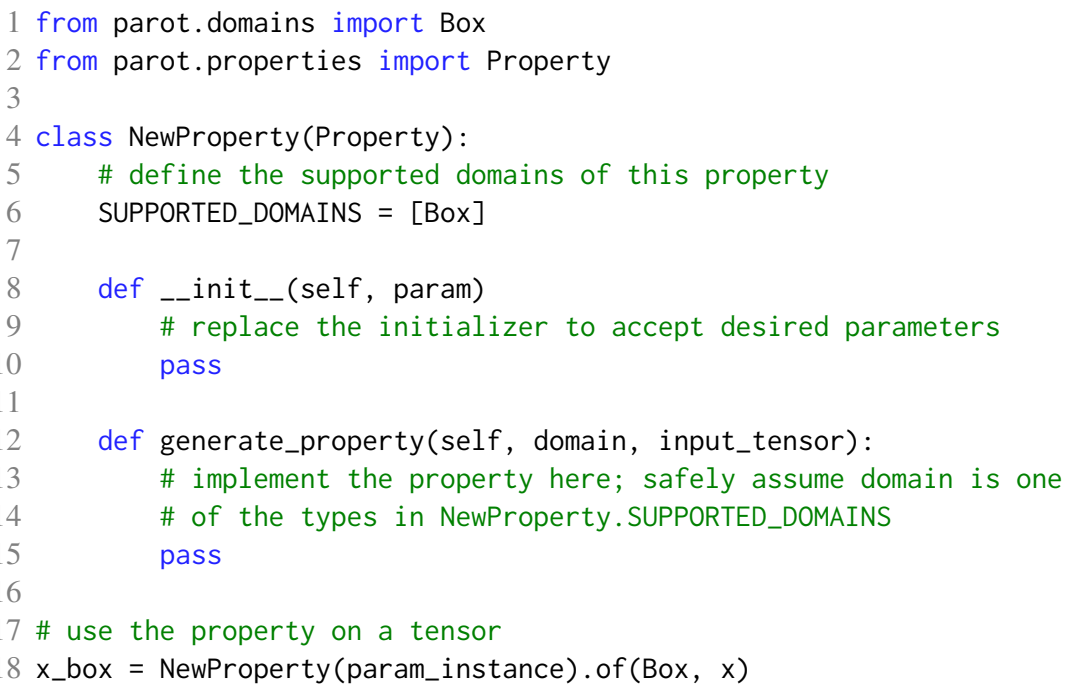

Listing 1.1. Implementing custom properties with PaRoT.

With these two considerations in mind, we have a procedure for transforming arbitrary TensorFlow graphs composed from a set of atomic transformers.

Figure 5 illustrates computing the transformed graph of the nodes on the left-hand side which represent the ops of a dense layer. Each green group on the right-hand side is the generated transformer computation graph of the corresponding $f$ in the domain HYBRIDZONOTOPE, i.e.,, the result of calling $\mathcal{D}(f)$ for the op. Note that the variables from the original layer are shared with the transformed ops.

\subsection{Robustness Properties}

In this section, we describe several built-in robustness properties that can be trained with in PaRoT, and an interface for specifying custom properties.

Built-in Properties Let $\mathbb{1}_{s}$ denote a tensor with shape $s$ with all elements being ones. All the following supported properties are centered on a training input $x$ with shape $s$.

- BallDemoted: the $\ell_{\infty}$-norm ball adversarial attack represented as an axis-aligned BoX where $\mathbf{b}=\epsilon \cdot \mathbb{1}_{s}$.

- BallPromoted: another $\ell_{\infty}$-norm ball adversarial attack represented in the $\mathbf{E}$ matrix of the HYBRIDZONOTOPE as $\mathbf{E}=\epsilon \cdot \operatorname{diag}\left(\mathbb{1}_{s}\right)$

- Brightness: a simple property with a single column in $\mathbf{E}$ where all pixels may have a constant added to them. That is, $\mathbf{E}=\epsilon \cdot \mathbb{1} \ldots s, 1$.

- UniformChannel: similar to Brightness except that each channel of the image is allowed to vary independently. 


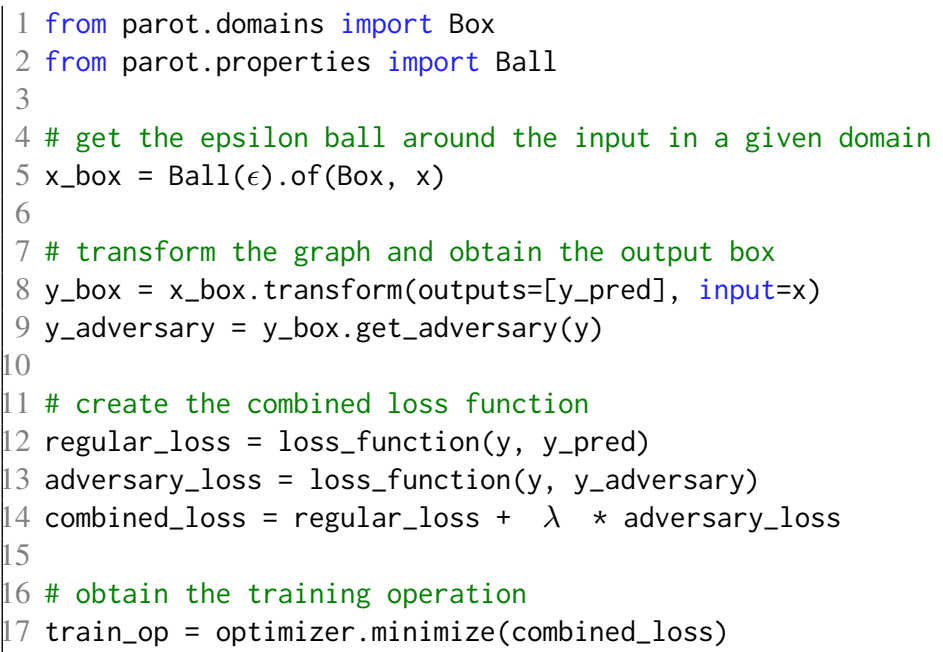

Listing 1.2. Given a model graph, return the training operation that optimizes a weighted version of the loss function for adversarial training.

- Fourier: for a 2D image $x$, each column of $\mathbf{E}$ is a plane wave. That is, each column of $\mathbf{E}$ is an image $I: H \times W \rightarrow \mathbb{R}$ :

$$
I(i, j)=\epsilon \cdot \kappa\left(i \frac{2 \pi n}{H}+j \frac{2 \pi m}{W}\right)
$$

for $\kappa \in\{\sin , \cos \}, n \in\{-N, \ldots, N\} \subset \mathbb{Z}$ and $m \in\{-M, \ldots, M\} \subset \mathbb{Z}$. Our motivation to investigate this property is to study the robustness to perturbations that we might observe in real data collected in the field. For example, in the case of detecting traffic lights, we can investigate whether it is possible to attack the network using only low frequencies (to model markings or distortions on a physical traffic light). An example of an adversarial example obtained through the Fourier on MNIST [24] is shown in Figure 6.

Custom Properties Defining a custom property in PaRoT is as simple as implementing a child class of Property, as presented in Listing 1.1.

\subsection{Robust Training using PaRoT}

Integrating our framework in a codebase can easily be done with minimal changes to the existing code, as exemplified in Listing 1.2. Given a training dataset with inputs $x$ and groundtruth outputs $y$ in tensor form, as well as the predictions of the model for the inputs, y_pred, we create a domain object using a BoX abstraction around the inputs and transform the resulting computation graph. Then, a combined loss function can be created and passed to the desired optimizer for robust training. 


\section{Experiments}

We evaluate PaRoT quantitatively to demonstrate performance, and qualitatively to validate its ease of use. We first show that our performance is comparable to the results obtained by DiffAI [27]. We then exemplify the ease of use on pre-trained models and finish with qualitative examples demonstrating a PaRoT robustness property. Throughout these experiments, we use the terms 'standard', 'regular' and 'baseline' interchangeably to describe a training process that solely uses a sparse cross-entropy loss.

In quantitative experiments, we make use of three metrics to measure performance:

- Test Error: percentage of misclassified examples in the testing set; the complement of classification accuracy.

- Test error under a PGD attack: a test based on the state-of-the-art Projected Gradient Descent attack first presented in [26] and used in [27]. PGD finds an adversarial example by following the gradient of the loss function inside an $\epsilon$-ball around the actual test example on the input side. Thus, the reported values correspond to a lower bound on the percentage of the misclassified examples in the testing set that are susceptible to an attack of this type.

- Test error under a HYBRIDZONOTOPE attack Verify: similar to the hSwi tch upper bound metric in [27], this metric uses the adversarial example discovered by the HYBRIDZONOTOPE on the output side, as in (11). Thus, the reported values correspond to an upper bound of the percentage of verifiably-susceptible examples in the testing set under this attack.

\subsection{DiffAI Comparison}

To validate the results of our framework, we ran robust training experiments similar to those in [27] of the BOX and HYBRIDZONOTOPE domains for MNIST [24] and CIFAR10 [23]. The architecture of the networks used is as defined in [27] and is also presented in Appendix B. We similarly augment the loss with an adversarial term with weight $\lambda=0.1$ and an $L 2$ regularization constant of 0.01 . The learning rate and $\epsilon$ used are $10^{-3}$ and 0.1 for MNIST, and $10^{-4}$ and 0.007 for CIFAR 10 , respectively. We run all experiments for 100 epochs using the Adam optimizer [21]. The results are in Table 1.

As the table shows, our framework achieves comparable results to those obtained in [27]. In all cases, introducing an adversarial training method leads to a minor drop in accuracy - an expected outcome when optimizing for a combined loss function with a finite capacity $[37,18]$. In terms of Box training, we observe, as expected, a slight increase in PGD and a strong increase in the number of verifiably-safe examples. For a HYBRIDZONOTOPE training when compared to the baseline, we notice that the number of examples susceptible to a PGD attack grows slightly while, in general, the number of verifiable cases improves significantly. Overall, these results are similar and in many cases improve upon the ones in [27] with minor exceptions that can be justified by implementation differences and stochasticity in weight initialization.

\subsection{Re-training Models}

In this experiment, we showcase the ease of use of PaRoT using a pre-trained network. We train a network with two convolutional layers and two dense layers, following the 
PaRoT: A Practical Framework for Robust Deep Neural Network Training

\begin{tabular}{|c|c|c|c|c|c|}
\hline Dataset & Model & Train Method & Test Error \% & PGD \% & Verify $\%$ \\
\hline \multirow{18}{*}{ MNIST } & \multirow{3}{*}{ FFNN } & Baseline & 1.8 & 3.2 & 100.0 \\
\hline & & Box & 3.2 & 4.2 & 30.6 \\
\hline & & HYBRIDZONOTOPE & 3.2 & 4.0 & 30.2 \\
\hline & \multirow{3}{*}{ ConvSmall } & Baseline & 1.4 & 2.4 & 100.0 \\
\hline & & Box & 2.0 & 2.4 & 12.8 \\
\hline & & HYBRIDZONOTOPE & 1.8 & 2.4 & 91.8 \\
\hline & \multirow{3}{*}{ ConvMed } & Baseline & 1.8 & 2.2 & 100.0 \\
\hline & & Box & 1.8 & 2.2 & 13.6 \\
\hline & & HYBRIDZONOTOPE & 2.4 & 2.6 & 88.6 \\
\hline & \multirow{3}{*}{ ConvBig } & Baseline & 0.6 & 1.2 & 100.0 \\
\hline & & Box & 1.2 & 1.4 & 14.0 \\
\hline & & HYBRIDZONOTOPE & 1.8 & 2.0 & 74.2 \\
\hline & \multirow{3}{*}{ ConvSuper } & Baseline & 0.6 & 1.0 & 100.0 \\
\hline & & Box & 1.0 & 1.2 & 12.2 \\
\hline & & HYBRIDZONOTOPE & 1.0 & 1.6 & 72.4 \\
\hline & \multirow{3}{*}{ Skip } & Baseline & 0.6 & 0.8 & 100.0 \\
\hline & & Box & 1.0 & 1.8 & 11.0 \\
\hline & & HYBRIDZONOTOPE & 0.8 & 1.6 & 10.0 \\
\hline \multirow{18}{*}{ CIFAR10 } & \multirow{3}{*}{ FFNN } & Baseline & 45.8 & 45.8 & 100.0 \\
\hline & & Box & 50.4 & 50.4 & 76.2 \\
\hline & & HYBRIDZONOTOPE & 48.8 & 48.8 & 75.8 \\
\hline & \multirow{3}{*}{ ConvSmall } & Baseline & 33.3 & 33.4 & 100.0 \\
\hline & & Box & 36.2 & 36.2 & 72.0 \\
\hline & & HYBRIDZONOTOPE & 38.6 & 38.6 & 96.2 \\
\hline & \multirow{3}{*}{ ConvMed } & Baseline & 34.6 & 34.6 & 100.00 \\
\hline & & Box & 35.8 & 35.8 & 69.6 \\
\hline & & HYBRIDZONOTOPE & 34.4 & 34.6 & 96.4 \\
\hline & \multirow{3}{*}{ ConvBig } & Baseline & 35.4 & 35.6 & 100.0 \\
\hline & & Box & 36.0 & 36.0 & 71.2 \\
\hline & & HYBRIDZONOTOPE & 38.0 & 38.0 & 99.4 \\
\hline & \multirow{3}{*}{ ConvSuper } & Baseline & 34.4 & 35.2 & 100.0 \\
\hline & & Box & 33.6 & 34.2 & 100.0 \\
\hline & & HYBRIDZONOTOPE & 35.3 & 35.4 & 98.6 \\
\hline & \multirow{3}{*}{ Skip } & Baseline & 34.0 & 34.6 & 100.0 \\
\hline & & Box & 40.0 & 39.8 & 73.2 \\
\hline & & HYBRIDZONOTOPE & 39.4 & 39.6 & 74.0 \\
\hline
\end{tabular}

Table 1. Quantitative Comparison: results of running our framework on the same datasets, architectures and parameters as in [27]. In the experiments run, we used $\epsilon=0.1$ for MNIST and 0.007 for CIFAR10. 


\begin{tabular}{l|ccc} 
Model & Test Error \% & PGD \% & Verify \% \\
\hline Original & 1.70 & 2.30 & 100.00 \\
Re-trained (Box) & 2.88 & 1.47 & 14.80
\end{tabular}

Table 2. Re-training Models: comparison between the original network trained only with standard loss and a re-trained network using an adversarial loss term.

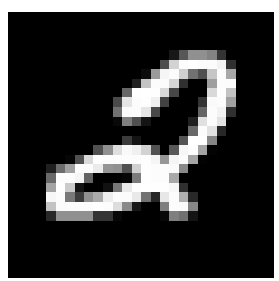

(a)

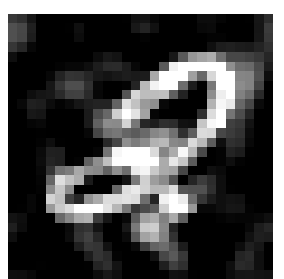

(b)

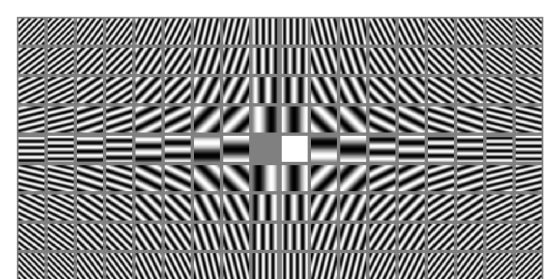

(c)

Fig. 6. Fourier Attack: example of a Fourier attack on an image of the MNIST dataset (a) an image that under regular training is correctly identified as a 2 (b) an adversarial example identified using PaRoT and a Fourier attack which leads the network trained with a standard loss function to identify as a 3. (c) is a grid of thumbnails of the available Fourier terms that were added to (a).

architecture of ConvSmall (see [27]), on the MNIST dataset using a standard loss for 200 epochs (learning rate of $10^{-3}$ ) and save it to a TensorFlow checkpoint file. We proceed to load this checkpoint's graph, and, using PaRoT's Box abstract domain, add an adversarial term to the loss function, which we then use to further train the loaded model for 100 epochs. The results of the process are presented in Table 2. Re-training achieves similar accuracy, while improving significantly the PGD and verification metrics. It should be noted that at no point in the re-training process did we have to re-define the model or state the required operations, one of the main advantages of our framework.

\subsection{Custom Robustness Properties: Case Study}

As described in Section 3.2, PaRoT includes a variety of built-in robustness properties on 2D images for HYBRIDZONOTOPE. In this section, we showcase the identification of adversarial examples based on the Fourier property. Figure 6 exemplifies an attack on a regularly trained network following the architecture of ConvMed (see [27]). The Fourier robustness property is motivated by the observation that a typical adversarial attack will include high frequency components which may be filtered away or rendered irrelevant by the variability in the real-world input image. It is interesting to ask whether adversarial examples exist only consisting of frequencies at roughly the scale of the original image.

In this scenario, the network correctly identifies the Figure 6a as a 2, yet is stumped by the adversarially generated image of Figure $6 \mathrm{~b}$ (using the Fourier terms presented in Figure 6c), mistakenly identifying it as a 3. After training with HYBRIDZONOTOPE with $\lambda=0.1$ and $\epsilon=0.01$ for 200 epochs for this robustness property, the model correctly identifies this specific example as a 2. 


\section{Related Work}

We consider three main areas of related work: early, heuristic approaches to training more robust networks; formal verification tools that typically operate on fully trained networks; and several other representative robust training approaches.

Heuristic approaches. Early art in adversarial robustness in the deep learning community broadly tackled the problem with heuristic techniques: with architecture and training scheme modifications $[14,44,6,38]$. These techniques have shown quite impressive results, and real progress has been made to training more inherently robust networks. However, it has been shown that these networks often remain susceptible to simple attacks [30,29]. This game of cat and mouse has led to ever more sophisticated attack and defense, e.g., $[12,36,43,15,26,10,2]$. In terms of their usability, however, many of these early approaches are comparable to ours. A lot of the techniques involve modifications to the training scheme rather than the network architecture itself. As has been shown, this is broadly similar to how robust training can be applied within our framework. The main limitation of these approaches is that they do not provide guarantees for robustness and, as ever, the bad guys tend to be one step ahead.

Formal verification Formal verification techniques provide guarantees on the robustness of a DNN at individual data-points [20,19,13,3,16], and, in at least one case for a small single-layer network, across the entirety of the input space [31]. Most of the work in this area focuses on Satisfiability Modulo Theories (SMT), reachability or optimization-based approaches to provide sound and complete guarantees on a perexample basis [25]. It has been shown that many of these techniques can be viewed as flavours of a unified Branch-and-Bound framework [5]. Through this lens, one can see the scalability challenges as an artifact of the combinatorial branching associated with piecewise-linear activation functions such as ReLUs. Similar to our framework, these formal verification tools require no modification to an existing codebase. In practice, most of the tooling is limited to a small subset of DNN activations and layer types (e.g., convolutional networks are often not supported) limiting their utility in practice. Furthermore, the intractability of these approaches, as detailed in [25], detracts their use in many of the larger networks we study in this paper. Lastly, as has been previously noted, these approaches do not offer systematic improvements at scale, i.e., the verification or falsification of each point needs to be considered iteratively in the training process.

Verifiably Robust Training Our work falls within a verifiably robust training approach. We omit any theoretical comparison of the approaches, which is well described in [27]. Our system is most closely similar to DiffAI [27]. However, it distinguishes itself in the way the abstract transformers are generated from existing models: DiffAI requires that the user specify their model using specialized classes. This makes their library difficult to use with pre-existing models, since it requires rewriting the models to fit within the DiffAI framework. Our framework, on the other hand, can take an existing TensorFlow graph [1] representing a model and transform it automatically without having to rewrite any model code, as shown in Section 3. This makes it more practical to use 
within an existing pipeline, as it decouples the maintenance of the model from the verified robustness procedure, allowing for faster development and testing. We observe that PaRoT achieves similar performance to DiffAI and in a similar total training times to those reported in [27] for the same GPU configuration (Nvidia GeForce GTX $1080 \mathrm{Ti}$ ).

In [39], the introduced framework, MixTrain, reaches better accuracy and a higher percentage of verifiably-safe examples when compared to [27]. Similarly, comparing the results presented in [39] with ours, we conclude that MixTrain outperforms the ones obtained in Section 4. However, it should be noted that some of techniques that MixTrain uses to achieve this improvement can be replicated easily when using our framework. For example, while in Listing 1.2 we defined the loss function as in [27] for the sake of simplicity, our framework allows for flexible definitions, including the dynamic loss function defined per epoch in [39].

Other works in this area involve convex relaxation techniques such as the ones presented in [33], or dual optimization techniques as in [42]. In terms of accuracy and adversarial robustness, further studies need to be carried out to compare our work to [33] and [42]. Despite this, the implementation of both [33] and [42] requires the rewriting of the models to adapt to the method's requirements, which, as in the case of [27], constitutes a set back to integration efforts in production software stacks.

\section{Conclusion and Future Work}

In this paper we introduce PaRoT, a novel framework for verifiable robust training that can be used directly on existing codebases and requires minimal code changes. We believe that this is the first practical framework for robust training that supports the vast majority of operations required for most large-scale models. Our work further contributes to the community with the introduction of new abstract transformers, novel formal robustness properties, and a framework for adding user-defined properties to robust training. We plan to build upon this framework in several directions:

- We wish to investigate more natural training schemes that, for example, use the robust loss more effectively and adapt the robustness property through the training cycle. Similarly, we plan to explore how we could provide features such as the stochastic robust approximation techniques from [39] for better performance.

- We also want to perform a theoretical study of abstract domains and training techniques that scale better with larger DNN widths and lengths; a fundamental problem of most of the methods presented in Section 5 [25]. It can be seen in Table 1, for example, that HYBRIDZONOTOPEs did not perform as well as would be expected on larger networks, and an in-depth analysis could help shed some light on the cause of this phenomenon.

- We wish to introduce an API for users to easily add and test their own op transformers, so that the framework can easily be extended to work on model code with currently unsupported ops. Currently supported ops may be viewed in Appendix A.

- Finally, we would like to conduct a comprehensive ablation study that includes many of the alternatives mentioned in Section 5 to further understand the comparative performance of our framework. 


\section{References}

1. Abadi, M., Barham, P., Chen, J., Chen, Z., Davis, A., Dean, J., Devin, M., Ghemawat, S., Irving, G., Isard, M., et al.: TensorFlow: A system for large-scale machine learning. In: 12th \{USENIX\} Symposium on Operating Systems Design and Implementation (\{OSDI\} 16). pp. 265-283 (2016)

2. Akhtar, N., Mian, A.S.: Threat of adversarial attacks on deep learning in computer vision: A survey. IEEE Access 6, 14410-14430 (2018)

3. Akintunde, M., Lomuscio, A., Maganti, L., Pirovano, E.: Reachability analysis for neural agent-environment systems. In: Principles of Knowledge Representation and Reasoning: Proceedings of the Sixteenth International Conference, KR 2018, Tempe, Arizona, 30 October - 2 November 2018. pp. 184-193 (2018)

4. Bojarski, M., Testa, D.D., Dworakowski, D., Firner, B., Flepp, B., Goyal, P., Jackel, L.D., Monfort, M., Muller, U., Zhang, J., Zhang, X., Zhao, J., Zieba, K.: End to end learning for self-driving cars. CoRR abs/1604.07316 (2016)

5. Bunel, R., Turkaslan, I., Torr, P.H.S., Kohli, P., Mudigonda, P.K.: A unified view of piecewise linear neural network verification. In: Advances in Neural Information Processing Systems 31: Annual Conference on Neural Information Processing Systems 2018, NeurIPS 2018, 3-8 December 2018, Montréal, Canada. pp. 4795-4804 (2018)

6. Cissé, M., Bojanowski, P., Grave, E., Dauphin, Y.N., Usunier, N.: Parseval networks: Improving robustness to adversarial examples. In: Proceedings of the 34th International Conference on Machine Learning, ICML 2017, Sydney, NSW, Australia, 6-11 August 2017. pp. 854-863 (2017)

7. Cousot, P., Cousot, R.: Abstract interpretation: a unified lattice model for static analysis of programs by construction or approximation of fixpoints. In: Proceedings of the 4th ACM SIGACT-SIGPLAN symposium on Principles of Programming Languages. pp. 238-252. ACM (1977)

8. Cousot, P., Cousot, R.: Abstract interpretation frameworks. Journal of Logic and Computation 2(4), 511-547 (1992)

9. Defense Advanced Research Projects Agency: Assured Autonomy, https://www.darpa.mil/ program/assured-autonomy

10. Dong, Y., Liao, F., Pang, T., Su, H., Zhu, J., Hu, X., Li, J.: Boosting adversarial attacks with momentum. In: 2018 IEEE Conference on Computer Vision and Pattern Recognition, CVPR 2018, Salt Lake City, UT, USA, June 18-22, 2018. pp. 9185-9193 (2018)

11. Goodfellow, I., Bengio, Y., Courville, A.: Deep Learning. The MIT Press (2016)

12. Goodfellow, I.J., Shlens, J., Szegedy, C.: Explaining and harnessing adversarial examples. In: 3rd International Conference on Learning Representations, ICLR 2015, San Diego, CA, USA, May 7-9, 2015, Conference Track Proceedings (2015)

13. Gopinath, D., Wang, K., Zhang, M., Pasareanu, C.S., Khurshid, S.: Symbolic execution for deep neural networks. CoRR abs/1807.10439 (2018)

14. Gu, S., Rigazio, L.: Towards deep neural network architectures robust to adversarial examples. In: 3rd International Conference on Learning Representations, ICLR 2015, San Diego, CA, USA, May 7-9, 2015, Workshop Track Proceedings (2015)

15. Huang, R., Xu, B., Schuurmans, D., Szepesvári, C.: Learning with a strong adversary. CoRR abs/1511.03034 (2015), http://arxiv.org/abs/1511.03034

16. Huang, X., Kwiatkowska, M., Wang, S., Wu, M.: Safety verification of deep neural networks. In: Majumdar, R., Kunvcak, V. (eds.) Computer Aided Verification. pp. 3-29. Springer International Publishing, Cham (2017)

17. Janai, J., Güney, F., Behl, A., Geiger, A.: Computer vision for autonomous vehicles: Problems, datasets and state-of-the-art. CoRR abs/1704.05519 (2017) 
18. Jetley, S., Lord, N.A., Torr, P.H.: With friends like these, who needs adversaries? In: Proceedings of the 32Nd International Conference on Neural Information Processing Systems. pp. 10772-10782. NIPS'18, Curran Associates Inc., USA (2018)

19. Katz, G., Barrett, C.W., Dill, D.L., Julian, K., Kochenderfer, M.J.: Reluplex: An efficient SMT solver for verifying deep neural networks. In: Computer Aided Verification - 29th International Conference, CAV 2017, Heidelberg, Germany, July 24-28, 2017, Proceedings, Part I. pp. 97-117 (2017)

20. Katz, G., Huang, D.A., Ibeling, D., Julian, K., Lazarus, C., Lim, R., Shah, P., Thakoor, S., Wu, H., Zeljić, A., Dill, D.L., Kochenderfer, M.J., Barrett, C.: The Marabou framework for verification and analysis of deep neural networks. In: Dillig, I., Tasiran, S. (eds.) Computer Aided Verification. pp. 443-452. Springer International Publishing, Cham (2019)

21. Kingma, D.P., Ba, J.: Adam: A method for stochastic optimization. arXiv preprint arXiv:1412.6980 (2014)

22. Koopman, P., Wagner, M.: Challenges in autonomous vehicle testing and validation (apr 2016)

23. Krizhevsky, A., et al.: Learning multiple layers of features from tiny images. Tech. rep., Citeseer (2009)

24. LeCun, Y., Bottou, L., Bengio, Y., Haffner, P., et al.: Gradient-based learning applied to document recognition. Proceedings of the IEEE 86(11), 2278-2324 (1998)

25. Liu, C., Arnon, T., Lazarus, C., Barrett, C., Kochenderfer, M.J.: Algorithms for verifying deep neural networks. arXiv preprint arXiv:1903.06758 (2019)

26. Madry, A., Makelov, A., Schmidt, L., Tsipras, D., Vladu, A.: Towards deep learning models resistant to adversarial attacks. In: 6th International Conference on Learning Representations, ICLR 2018, Vancouver, BC, Canada, April 30 - May 3, 2018, Conference Track Proceedings (2018)

27. Mirman, M., Gehr, T., Vechev, M.: Differentiable abstract interpretation for provably robust neural networks. In: Dy, J., Krause, A. (eds.) Proceedings of the 35th International Conference on Machine Learning. Proceedings of Machine Learning Research, vol. 80, pp. 3578-3586. PMLR, Stockholmsmssan, Stockholm Sweden (10-15 Jul 2018)

28. Mirman, M., Singh, G., Vechev, M.T.: A provable defense for deep residual networks. CoRR abs/1903.12519 (2019)

29. Moosavi-Dezfooli, S., Fawzi, A., Fawzi, O., Frossard, P.: Universal adversarial perturbations. In: 2017 IEEE Conference on Computer Vision and Pattern Recognition (CVPR). pp. 86-94 (July 2017). https://doi.org/10.1109/CVPR.2017.17

30. Papernot, N., McDaniel, P., Jha, S., Fredrikson, M., Celik, Z.B., Swami, A.: The limitations of deep learning in adversarial settings. In: 2016 IEEE European Symposium on Security and Privacy (EuroS P). pp. 372-387 (March 2016)

31. Raghunathan, A., Steinhardt, J., Liang, P.: Certified defenses against adversarial examples. In: 6th International Conference on Learning Representations, ICLR 2018, Vancouver, BC, Canada, April 30 - May 3, 2018, Conference Track Proceedings (2018)

32. Salay, R., Czarnecki, K.: Using machine learning safely in automotive software: An assessment and adaption of software process requirements in ISO 26262. CoRR abs/1808.01614 (2018)

33. Salman, H., Yang, G., Zhang, H., Hsieh, C.J., Zhang, P.: A convex relaxation barrier to tight robustness verification of neural networks. arXiv preprint arXiv:1902.08722 (2019)

34. Singh, G., Gehr, T., Mirman, M., Püschel, M., Vechev, M.: Fast and effective robustness certification. In: Advances in Neural Information Processing Systems. pp. 10802-10813 (2018)

35. Szegedy, C., Zaremba, W., Sutskever, I., Bruna, J., Erhan, D., Goodfellow, I.J., Fergus, R.: Intriguing properties of neural networks. In: 2nd International Conference on Learning Representations, ICLR 2014, Banff, AB, Canada, April 14-16, 2014, Conference Track Proceedings (2014) 
36. Tramèr, F., Kurakin, A., Papernot, N., Goodfellow, I.J., Boneh, D., McDaniel, P.D.: Ensemble adversarial training: Attacks and defenses. In: 6th International Conference on Learning Representations, ICLR 2018, Vancouver, BC, Canada, April 30 - May 3, 2018, Conference Track Proceedings (2018)

37. Tsipras, D., Santurkar, S., Engstrom, L., Turner, A., Madry, A.: Robustness may be at odds with accuracy. In: 7th International Conference on Learning Representations, ICLR 2019, New Orleans, LA, USA, May 6-9, 2019 (2019)

38. Wang, B., Gao, J., Qi, Y.: A theoretical framework for robustness of (deep) classifiers against adversarial samples. In: 5th International Conference on Learning Representations, ICLR 2017, Toulon, France, April 24-26, 2017, Workshop Track Proceedings (2017)

39. Wang, S., Chen, Y., Abdou, A., Jana, S.: Mixtrain: Scalable training of formally robust neural networks. arXiv preprint arXiv:1811.02625 (2018)

40. Wang, X., Yu, F., Dou, Z.Y., Darrell, T., Gonzalez, J.E.: Skipnet: Learning dynamic routing in convolutional networks. In: Proceedings of the European Conference on Computer Vision (ECCV). pp. 409-424 (2018)

41. Wong, E., Kolter, J.Z.: Provable defenses against adversarial examples via the convex outer adversarial polytope. In: Proceedings of the 35th International Conference on Machine Learning, ICML 2018, Stockholmsmässan, Stockholm, Sweden, July 10-15, 2018. pp. 5283$5292(2018)$

42. Wong, E., Schmidt, F., Metzen, J.H., Kolter, J.Z.: Scaling provable adversarial defenses. In: Advances in Neural Information Processing Systems. pp. 8400-8409 (2018)

43. Yuan, X., He, P., Zhu, Q., Li, X.: Adversarial examples: Attacks and defenses for deep learning. IEEE Trans. Neural Netw. Learning Syst. 30(9), 2805-2824 (2019)

44. Zheng, S., Song, Y., Leung, T., Goodfellow, I.: Improving the robustness of deep neural networks via stability training. In: 2016 IEEE Conference on Computer Vision and Pattern Recognition (CVPR). pp. 4480-4488 (June 2016) 


\section{Appendix A Implemented TensorFlow Operations and Keras Layers}

Table 3 lists the currently implemented TensorFlow operations in PaRoT, while Table 4 shows the implemented Keras layers. Other Keras layers might be supported depending on the implementation in terms of TensorFlow operations.

\section{Appendix B Network architectures}

We follow the design of [27]. For convolutional layers $c \times w \times h[s]$ is for channels, kernel width, kernel height and stride, respectively.

FFNN Five fully-connected layers, 100-node each, with ReLU.

ConvSmall Two convolutional layers with no padding $(16 \times 4 \times 4$ [2], $32 \times 4 \times 4$ [2]), followed by a 100-node fully-connected layer.

ConvMed Two convolutional layers with padding of $1(16 \times 4 \times 4$ [2], $32 \times 4 \times 4$ [2]), followed by a 100-node fully-connected layer.

ConvBig Four convolutional layers with padding of 1 ( $32 \times 3 \times 3$ [1], $32 \times 4 \times 4$ [2], $64 \times 3 \times 3$ [1], $64 \times 4 \times 4$ [2]), followed by a 512-node fully-connected layer, ReLU, and a 512-node fully-connected layer.

ConvSuper Four convolutional layers with no padding ( $32 \times 3 \times 3$ [1] , $32 \times 4 \times 4$ [1], $64 \times 3 \times 3$ [1], $64 \times 4 \times 4$ [1]), followed by a 512-node fully-connected layer, ReLU, and a 512-node fully-connected layer.

Skip A concatenation of two covolutional networks followed by ReLU, 200-node fullyconnected network, and ReLU. The two networks are:

- Three convolutional layers $(16 \times 3 \times 3$ [1], $16 \times 3 \times 3$ [1], $32 \times 3 \times 3$ [1]), followed by a 200 -node fully-connected layer

- Two convolutional layers $(32 \times 4 \times 4$ [1], $32 \times 4 \times 4$ [1]) followed by a 200-node fully-connected layer. 


\begin{tabular}{|c|c|c|}
\hline Operation Type & Box & HYBRIDZONOTOPE \\
\hline Abs & & $\checkmark$ \\
\hline Add & $\sqrt{ }$ & $\checkmark$ \\
\hline BiasAdd & $\checkmark$ & $\sqrt{ }$ \\
\hline ConcatV2 & $\checkmark$ & $\begin{array}{c}\sqrt{\text { (only between } \mathrm{HZ} \text { and }} \\
\text { tf.Tensor) }\end{array}$ \\
\hline Conv2D & $\sqrt{ }$ & $\begin{array}{c}\sqrt{\text { (the second input should }} \\
\text { be tf.Tensor) }\end{array}$ \\
\hline Exp & & $\checkmark$ \\
\hline GreaterEqual & $\sqrt{ }$ & $\sqrt{ }$ \\
\hline Log & & $\checkmark$ \\
\hline $\log 1 p$ & & $\checkmark$ \\
\hline MatMul & $\sqrt{ }$ & $\checkmark$ (only first input HZ) \\
\hline Maximum & & $\checkmark$ \\
\hline MaxPool & $\checkmark$ & $\begin{array}{c}\checkmark(\text { only for } \\
\text { kkeras.MaxPool2D(2)) }\end{array}$ \\
\hline Mean & & $\checkmark$ \\
\hline Minimum & & $\checkmark$ \\
\hline Mul & $\checkmark$ & $\sqrt{ }$ \\
\hline Neg & $\checkmark$ & $\checkmark$ \\
\hline OnesLike & $\sqrt{ }$ & $\sqrt{ }$ \\
\hline Pack & $\checkmark$ & \\
\hline RealDiv & $\checkmark$ & $\sqrt{ }$ \\
\hline Relu & $\sqrt{ }$ & $\sqrt{ }$ \\
\hline Reshape & $\sqrt{ }$ & $\sqrt{ }$ \\
\hline Select & & $\checkmark$ (first input not HZ) \\
\hline Shape & $\checkmark$ & $\checkmark$ \\
\hline Sigmoid & $\sqrt{ }$ & $\checkmark$ \\
\hline Softmax & $\checkmark$ & $\sqrt{ }$ \\
\hline StridedSlice & $\checkmark$ & $\checkmark$ \\
\hline Sub & $\checkmark$ & $\checkmark$ \\
\hline Sum & & $\checkmark$ \\
\hline Transpose & $\checkmark$ & $\checkmark$ \\
\hline ZerosLike & $\checkmark$ & $\sqrt{ }$ \\
\hline
\end{tabular}

Table 3. TensorFlow operations implemented in PaRoT 


\begin{tabular}{l|cc} 
Layer & BOX & HYBRIDZONOTOPE \\
\hline Concatenate & $\checkmark$ & $\checkmark$ \\
\hline Conv2D & $\checkmark$ & $\checkmark$ \\
\hline Dense('relu') & $\checkmark$ & $\checkmark$ \\
\hline Dense('sigmoid') & $\checkmark$ & $\checkmark$ \\
\hline Dense('softmax') & $\checkmark$ & $\checkmark$ \\
\hline Flatten & $\checkmark$ & $\checkmark$ \\
\hline MaxPooling2D & $\checkmark$ & $\checkmark$ \\
\hline
\end{tabular}

Table 4. Keras layers supported by PaRoT out of the box 